\title{
Biological Engineering Analysis of Vermicompost Based on Image Features and Machine Learning
}

\author{
Hongyan Wang $\mathbb{D}^{1},{ }^{1}$ Ling Wang $\mathbb{D}^{1},{ }^{1}$ Jiabin Liu $\mathbb{D}^{2},{ }^{2}$ Ying Nie $\mathbb{D}^{3},{ }^{3}$ and Daqing Wang $\mathbb{D}^{2,3}$ \\ ${ }^{1}$ College of Resources and Environment, Northeast Agricultural University, Harbin 150030, China \\ ${ }^{2}$ Beidahuang Strategy and Economic Development Research Center, Agricultural Reclamation Management and Cadres College, \\ Harbin 150090, China \\ ${ }^{3}$ Faculty of Foreign Trade and Toreign Languages, Haikou University of Economics, Haikou 571127, China \\ Correspondence should be addressed to Daqing Wang; wangdaqing511@163.com
}

Received 3 November 2021; Revised 14 December 2021; Accepted 17 December 2021; Published 3 January 2022

Academic Editor: Sang-Bing Tsai

Copyright (c) 2022 Hongyan Wang et al. This is an open access article distributed under the Creative Commons Attribution License, which permits unrestricted use, distribution, and reproduction in any medium, provided the original work is properly cited.

\begin{abstract}
Earthworm manure is a soil enhancement product that is homogeneous, permeable, ecological, and organic. It has a particle structure that is substantially greater than the soil's surface area. Using a suitable quantity of earthworm fertilizer in the soil will improve the nutritional state of the soil surface, as well as the microbial control system and drainage capacity. Bioengineering earthworm dung is now a tough challenge, but picture quality evaluation can help enhance the organic fertilizer treatment process for earthworm manure. Researchers began researching appropriate assessment methods in order to assure the influence of earthworm excrement and to precisely and effectively measure changes in image quality. As a result, we must first determine the consistency qualities, extract the image's color and texture, and then create a comparable vector with 11 dimensions. Finally, we learn how to train the picture quality regression model using the mechanical learning (ML) approach. As a result, an effective and precise image quality evaluation system was created, and earthworm manure bioengineering was effectively applied.
\end{abstract}

\section{Introduction}

Organic soil improvement is widely utilized for environmental restoration and is thought to be the most effective way for controlling soil plant disease. Earthworm excrement is a by-product of the reproduction of earthworms, and it is also an excellent organic fertilizer. Organic waste is a type of green organic fertilizer produced by enzyme fermentation in the digestive system of earthworms. It has been the topic of substantial research in the chemical and biological sciences in recent years due to its physical features, which are notably different from regular organic fertilizer products. The application of this high-quality fertilizer to plant diseases management is a significant innovation in the utilization of earthworm manure. The usage of earthworm manure can not only inhibit diseases but more importantly reduce the resistance of pathogens to chemicals. Therefore, the use of earthworm manure for biological prevention provides a strategy for the prevention and control of pathogen resistance. In terms of soil environmental management, the transformation, and action mechanism of various forms of soil nutrients, the research on earthworm manure is weak. A deeper understanding of the fertilization mechanism of earthworm manure can activate soil nutrients and help us better understand and use these bioengineered products, which can successfully address environmental, economic, and societal issues. The existing bioengineering of earthworm manure has some limitations. Image quality assessment can be used to improve the judgment of the earthworm manure organic fertilizer treatment process to ensure the effect of organic fertilizer. Machine learning is the data analysis method, which is used to analyze the model building automatically. ML is one of the branches of artificial intelligence used to determine patterns and data and take decision with less human interference. In agriculture, the machine learning is used to increase the quality and 
productivity of the crops. It is also utilized to determine different kinds of bugs, vermin, and bacteria's present in the soil. The evaluation of image quality is an important part of processing research. In recent years, with the development of information and communication technology, the use of smart terminals has rapidly become popular, and the application and demand for digital images have rapidly increased. Digital images have been widely used in many fields, including communications, biomedicine, industrial production, geological prospecting, weather forecasting, military defense, and space exploration. Image quality has an absolute impact on the value of applications. High-quality images are more useful for receiving, understanding, and processing image information. Due to physical environment and technical limitations, they are not available at all stages of image acquisition, transmission, and storage. It can avoid causing image distortion and poor image quality, which affects the image extraction. To accurately and effectively measure the changes in image quality, researchers began to study effective quality evaluation methods.

At present, some progress has been made in bioengineering research on earthworm excrement. Joshi et al. [1] found that earthworms may increase the rate of mineralization and degradation of organic waste during the biological oxidation stage. The basic plant nutrients in raw materials, such as nitrogen, potassium, phosphorus, and calcium, become more readily available forms than base plants. At the same time, most organic raw materials are decomposed into stable materials and converted into components similar to those in the soil. Gopalakrishnan et al. [2] showed that earthworms release body cavity fluid into fertilizers, which kill parasites and bacteria in waste materials, so that pathogens and the smell of earthworm feces disappear. Therefore, the earthworm composting technology is suitable for the reuse of organic waste because of its high cost-effectiveness. Arancon et al. [3] pointed out that some earthworms bear huge environmental pressure in the soil; they have strong organic decomposition ability, can highly consume and absorb organic matter, and hatch a lot through production and have a high reproduction rate. Pathma et al. [4] showed that a large number of microbial antagonists were found in earthworm excrement, and some resistant varieties have been isolated from general organic fertilizers. Antagonistic fungi dominate in mud excrement, while, in organic fertilizers, the antagonistic number of fungi is small. Simsek-Ersahin et al. [5] studied the abundance of bacteria and the structural changes caused by the application of these technologies. Biological fertilizer is a key factor to prevent the production of Fusarium oxysporum. In particular, the usage of biological fertilizers can increase the number of antibacterial communities, and they are controlling this. Pathogenic bacteria can play a key role.

According to image characteristics, mechanical learning is used to evaluate image quality. Prabal et al. [6] pointed out that the subjective evaluation results of image quality are very accurate and reliable, which are closer to humans' actual perceptions of image quality, and provide an important research foundation for objective evaluation of image quality. The subjective experimental design according to the ITU-RBT.500-13 standard is often used to image quality. These standards are proposed by the International Telecommunication Union. Renuka et al. [7] analyzed the relationship between the inherent geometric characteristics of the image and the visual characteristics, which is a natural phenomenon, and proposed a complete reference image quality evaluation method based on the average geometric model, which combines gradient and geometric characteristics. Rumsfeld et al. [8] showed that the objective evaluation of image quality usually includes designing specific mathematical models and mathematical formulas to evaluate the quality of the image and get results consistent with the evaluation. The objective evaluation of image quality will continue to be explored and researched. Sanjay et al. [9] proposed an evaluation method. The quality evaluation of a complete reference image is based on the similarity of characteristics. The information evaluation method about the image phase and gradient consistency (FSIM) and the color image quality evaluation method are based on color space (ICSC). Stergiou et al. [10] found that the comparable information about image brightness is an important factor affecting image quality. According to the assumption that the human visual system is more sensitive to image structure information, a FR-IQA method of structural similarity is proposed. The information characteristics of the structure, the brightness characteristic information, and the symbol information are combined. Alencar et al. [11] converted the image into a small waveband based on the statistical characteristics of the image and reflected the image quality by calculating the KL distance between the possible distribution of the small waveband. Alsarhan et al. [12] analyzed the human visual system, and the degree of distortion of the visual observation mechanism on the visible part of the image and a reference image quality evaluation method based on content distortion were proposed by Ammar et al. [13]. In the survey process, it is necessary to measure the relationship between accuracy and evaluation characteristics, and it is necessary to frequently write design research reports for specific situations.

In summary, a machine learning method is employed to learn the regression model in the image drawing stage, and a complete reference image quality evaluation method based on RF is proposed. Machine learning techniques are being used to replace traditional picture quality drawing methods. First, the phase, marginalization, color characteristics, and image texture characteristics are authenticated, and then 11 vectors with similar dimensional characteristics are found. Finally, through learning and training, we get a quality regression model, analyze the performance differences between various regression tools, and cross-check the database.

The authors of this paper firstly adopt the characteristics of phase consistency, frame consistency, color consistency, and image texture consistency. Then, we establish a similar vector with 11 dimensions, use the mechanical learning method to learn and train the image quality regression model for designing an effective and accurate image quality assessment method, and successfully carry out the earthworm fertilizer bioengineering. 


\section{Application of RF-IQA Algorithm in Full-Reference Image Quality Evaluation}

2.1. Visual Perception of Brightness Characteristics. From the perspective of the nature of visual brightness, we all know that the human visual system is not sensitive to the perception of absolute brightness of objects, and it is easier to perceive changes in the relative brightness of objects [14]. The human visual system's perception of object brightness depends on relative brightness. Visual perception is nothing but the ability of the brain to act, receive, and interrupt based on visual stimuli. Also, the patterns, structures, and colors of the specific interest relation in GUI are based on complete vision. It enables the skills of the designer to design more effective interfaces for users $[15,16]$. However, due to the limited resolution of the human visual system, when the difference between the target and the background is lower than a certain threshold, the perception of the brightness of the target and the background is the same, the so-called relative sensitivity, and Weber and Michelson are often used. The Michelson contrast is mainly used for patterns with both dark and bright features with equivalent and similar fractions of area. It is also defined to represent the lowest and highest luminance. Likewise, the term Weber contrast is used to define the relationship between luminance with the adjacent darker area and the luminance with the brighter area. In terms of this, the differences between luminance and another are separated with the help of lower luminance, expressing relative sensitivity.

The calculation formula for Weber comparison is as follows:

$$
C_{w}=\frac{\Delta L}{L}
$$

Calculate the subjective brightness of the target object:

$$
\begin{aligned}
\Delta S & =K \frac{\Delta I}{I} . \\
S_{w} & =K^{\prime} \log I+K_{0} .
\end{aligned}
$$

Michelson contrast calculation formula is shown in the following formula:

$$
C_{m}=\frac{L_{\max }-L_{\min }}{L_{\max }+L_{\min }} .
$$

Subjective brightness perception of the human visual system:

$$
S_{m}=k \ln B+k_{0} .
$$

There is no direct linear relationship between the subjective view of the brightness of an object in the human visual system and the actual objective brightness of the object. This can be seen from the above-mentioned Weber and Wilson comparison formula [17]. The visual system adapts to a variety of brightness according to its sensitivity to light, which expresses the ability of the human visual system to distinguish the brightness of an image. Use the CampbellRobson diagram to show this visual view, as shown in Figure 1. It is a chart, which is widely used to represent the

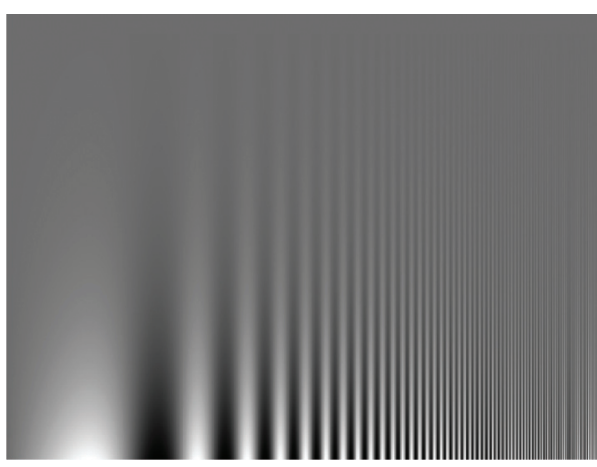

Figure 1: Campbell-Robson diagram.

visual perception textbooks and psychophysics based on the contrast sensitivity function (CSF). Also, it delivers the grating differs from the contrast from bottom to top and in spatial frequency (SF) from left to right logarithmically. This is a sample of comparative sensitivity [18]. Formula (6) is as follows:

$$
A(f)=2.6(00.192+0.114 f) e^{\left[-(0.114 f)^{1.1}\right]} .
$$

The results show that the human visual system is very sensitive to the area in the middle of the image signal and is less sensitive to high-frequency and low-frequency areas.

\subsection{Image Quality-Related Feature Selection}

2.2.1. Phase Consistency Characteristics. The Log-Gabor filter is further improved by the combination of a doublelayer filter Log-Gabo and Gabor [19]. The Gabor function is similar to the visual response to perception in the human visual system. The Gabor filter is a bandpass filter, which is mainly used in image processing for texture and features extraction by generating a simple sinusoidal form. With the help of the Gabor filter, the function orientation, size, and frequency are easily calculated to generate receptive field models with different ranges. Information about image space and frequency can be effectively extracted and often used as an image processing area. The Log-Gabor filter is the type of filter that is used to explain the signal in terms of local frequency signals. The Log-Gabor filter is the improved version of the Gabor filter. It is used to describe the corners of the image in terms of localized frequency information. The major advantage of the filter is to compare the images with Gabor filters and other wavelet filters. The limit of the Gabor filter is expanded to one-eighth, and there is no advantage in processing bandwidth information. Log-Gabor filter has no advantage compared with Gabor filter, and the bandwidth of Gabor filter can be determined arbitrarily, with additional expansion and coverage advantage; the formula used is as follows:

$$
G(w)=\exp \left(-\frac{\log \left(w / w_{0}\right)^{2}}{2 \sigma_{t}^{2}}\right) .
$$

The two-dimensional Log-Gabor filter is obtained by extending the one-dimensional Log-Gabor filter in the 
vertical direction. The transfer function of the filter is as follows:

$$
G_{2 D}(w, \theta)=\exp \left(-\frac{\log \left(w / w_{0}\right)^{2}}{2 \sigma_{t}^{2}}\right) \times \exp \left(-\frac{(\Omega-\theta)^{2}}{2 \sigma_{\theta}^{2}}\right) .
$$

Among them,

$$
\begin{aligned}
A_{S, \theta}(x) & =\sqrt{e_{s, \theta}(x)^{2}+o_{s, \theta}(x)^{2}}, \\
E_{\theta}(x) & =\sqrt{E_{\theta}(x)^{2}+H_{\theta}(x) 2}, \\
F_{\theta}(x) & =\sum_{s} e_{s, \theta}(x), H_{\theta}(x)=\sum_{s} o_{s, \theta}(x) .
\end{aligned}
$$

2.2.2. Edge Features. The edge feature is considered as the basic features of an image; it also defines image region boundaries by detecting pixel value discontinuities sharply by means of object recognition and segmentation. For the image IMG $(\mathrm{x})$, the gradient amplitudes in the $x$ direction and $y$ direction are shown as follows:

$$
\begin{aligned}
& \operatorname{GM}_{x}(x)=\operatorname{IMG}(x) * H_{x}, \\
& \operatorname{GM}_{y}(x)=\operatorname{IMG}(x) * H_{y} .
\end{aligned}
$$

The corresponding image gradient amplitude is shown in equation (12), and the gradient direction is shown in equation (13):

$$
\begin{aligned}
\mathrm{GM}(x) & =\sqrt{\mathrm{GM}_{x}(x)^{2}+\mathrm{GM}_{y}(x)^{2}}, \\
\theta(x) & =\arctan \left(\frac{\mathrm{GM}_{x}(x)}{\mathrm{GM}_{y}(x)}\right) .
\end{aligned}
$$

2.2.3. Color Characteristics. This article uses an HSV color space that is more in line with the color perception of the visual system [20]. It reprojects the RGB model through a cylindrical coordinate system, which is a more intuitive expression of color than the RGB model based on the geographic coordinate system [21]. The cylindrical coordinate system is the $3 \mathrm{D}$ coordinate system that indicates the position of the particular point by its distance from the reference axis. So, the relative axis direction is selected in terms of reference direction. Also, the distance from the reference plane perpendicular is selected from the axis of the direction, and the geographic coordinate system is the method, which is used to elucidate geographic location position on the surface of the Earth using longitude and latitude spherical measures. The angles are measured from the Earth point center in the Earth's surface [22, 23]. The color is represented by the color spectrum $(\mathrm{H})$, saturation $(\mathrm{S})$, and brightness (V). The basic characteristic of Saturation represents the purity of the color. The term HSV is the abbreviated form of Hue, Saturation, and Value. The HSV model is used to define the color space based on three major components such color type, hue, and range. The HSV is used by the designers to select the colors for ink or paint, because it represents the relation between the people and colors rather than the RGB color model. With the help of the HSV color wheel, the designer can generate highquality graphics [24]. The HSV model corresponds to a subset of cylinders on cylindrical coordinates, as shown in Figure 2.

The conversion formula of the HSV color model is as follows:

$$
H= \begin{cases}0^{*}, & \text { if } \max =\min , \\ 60^{*} \times \frac{G-B}{\max -\min }+0^{*}, & \text { if } \max =\operatorname{Rand} G \geq B, \\ 60^{*} \times \frac{G-B}{\max -\min }+360^{*}, & \text { if } \max =\operatorname{Rand} G<B, \\ 60^{*} \times \frac{B-R}{\max -\min }+120^{*}, & \text { if } \max =G, \\ 60^{*} \times \frac{R-G}{\max -\min }+240^{*}, & \text { if } \max =B, \\ S=\frac{\max -\min }{\max \times \frac{100}{255},} & \max \times \frac{100}{255} .\end{cases}
$$

2.2.4. Texture Features. Text features contain information of all components present in the article excluding the major body of the text. The text feature involves a glossary, bold words, index, and table of contents, sidebars, captions, and headings of the labeled diagram. Some studies have shown that the results of using the Scientific (SCI) method to measure complex texture conform to the cognitive characteristics of the human visual system and the complexity of the human visual system. SCI is the relationship between relative strength and structural strength. The calculation formula is as follows:

$$
\mathrm{SCI}=\frac{\mathrm{CI}^{\alpha}}{\mathrm{KT}^{\beta}}
$$

KT represents the randomness of the material model, which can be used to describe the background texture structure of the image, and is expressed by the integrated peak value of the Discrete Cosine Transform (DCT) area exchange coefficient as follows: 


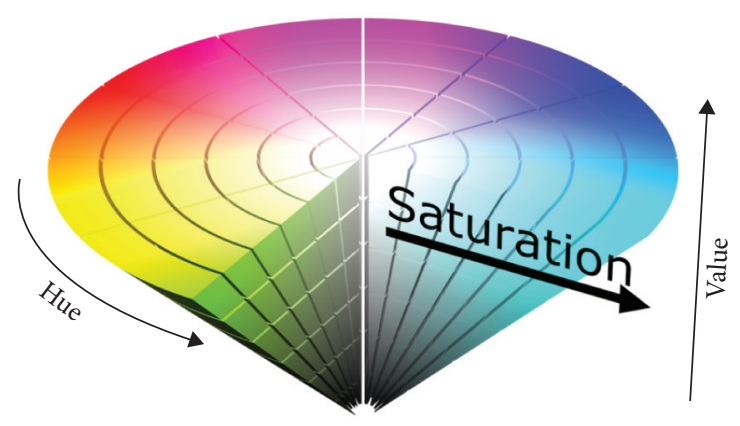

FIgURE 2: HSV color space model.

$$
\begin{aligned}
\mathrm{CI} & =\frac{m_{0}}{N^{2}}, \\
\mathrm{KT} & =\frac{m_{4}}{m_{2}^{2}}, \\
m_{k} & =\sum_{\omega \in B, \omega \notin 0} \omega^{k} p(\omega), \\
\omega & =\delta \cdot \sqrt{u^{2}+v^{2}}, \\
p(\omega) & =\frac{\left(\varepsilon+|c(\omega)|^{\lambda}\right)}{Z}, \\
Z & =\sum_{\omega \in B, \omega \notin 0}\left(\varepsilon+|c(\omega)|^{\lambda}\right) .
\end{aligned}
$$

In summary, we can obtain the calculation formula of SCI-1 as follows:

$$
\mathrm{SCI}^{-1}=\frac{\sum_{(u, v) \in A}\left\{\left(u^{2}+v^{2}\right)^{2} \cdot(\varepsilon+|c(u, v)|)\right\}}{\sum_{(u, v) \in A}\left\{\left(u^{2}+v^{2}\right)^{2} \cdot(\varepsilon+|c(u, v)|)\right\}^{2}} .
$$

2.3. Feature Similarity Calculation. The feature-based similarity is used to measure the characteristics and common features of the objects during comparison. It is also used to explore semantic information based on hierarchy-based approaches. When calculating the similarity between the phase consistency characteristics, the frame characteristics, and the texture characteristics, the color image will be converted into an equivalent luminescence image through the luminescence as follows:

$$
Y=0.3 R+0.59 G+0.11 B
$$

This is the effective brightness value of each pixel in the image, and $R, G$, and $B$ represent the similarity of the color brightness of the red, green, and blue channels of the color image, respectively:

$$
\begin{gathered}
S_{H}(x)=\frac{2 H_{I M G_{r}}(x) H_{I M G_{1}}(x)+\varepsilon_{1}}{H_{I M G_{r}}^{2}(x)+H_{I M G_{r}}^{2}(x)+\varepsilon_{1}}, \\
S_{S}(x)=\frac{2 S_{I M G_{r}}(x) S_{I M G_{1}}(x)+\varepsilon_{2}}{S_{I M G_{r}}^{2}(x)+S_{I M G_{r}}^{2}(x)+\varepsilon_{2}} .
\end{gathered}
$$

Phase consistency feature similarity:

$$
S_{P C}(x)=\frac{2 P C_{I M G_{r}}(x) P C_{I M G_{1}}(x)+\varepsilon_{3}}{P C_{I M G_{r}}^{2}(x)+P C_{I M G_{1}}^{2}(x)+\varepsilon_{3}} .
$$

Edge feature similarity:

$$
\begin{aligned}
S_{G M}(x) & =\frac{2 G M_{I M G_{r}}(x) G M_{I M G_{1}}(x)+\varepsilon_{4}}{G M_{I M G_{r}}^{2}(x)+G M_{I M G_{r}}^{2}(x)+\varepsilon_{4}} . \\
S_{\theta}(x) & =\frac{2 \theta_{I M G_{r}}(x) \theta_{I M G_{1}}(x)+\varepsilon_{5}}{\theta_{I M G_{r}}^{2}(x)+\theta_{I M G_{r}}^{2}(x)+\varepsilon_{5}} .
\end{aligned}
$$

Texture feature similarity:

$$
S_{S C I^{-1}}=\frac{2 S C I_{I M G_{r}}^{-1}(x) S C I_{I M G_{r}}^{-1}(x)+\varepsilon_{6}}{\left(S C I_{I M G_{r}}^{-1}\right)^{2}+\left(S C I_{I M G_{r}}^{-1}\right)^{2}+\varepsilon_{6}} .
$$

In summary, the mean value and standard deviation of the extracted feature similarity map together constitute an 11-dimensional feature vector $f$, as shown in the following formula:

$f=\left[u_{H}, \sigma_{H}, u_{s}, \sigma_{s}, u_{P C}, \sigma_{P C}, u_{G M}, \sigma_{G M}, u_{\theta}, \sigma_{\theta}, S_{S C I^{-1}}\right]$.

2.3.1. Image Quality Score Mapping Training Based on Machine Learning. As shown in the following formulas,

$$
\begin{gathered}
\min \frac{1}{2}\|\omega\|^{2}+C \sum_{i=1}^{m} l_{\varepsilon}\left(Y\left(x^{j}\right)-Q^{i}\right) \\
l_{\varepsilon}= \begin{cases}0, & |z| \leq \varepsilon, \\
|z|-\varepsilon, & \text { other, }\end{cases} \\
Y(x)=\sum_{i=1}^{m}\left(\widehat{a}_{i}-a_{i}\right) K\left(x_{i}, x\right)+b .
\end{gathered}
$$

In the vector regression support model, it is carried out by using a characteristic similar vector and the corresponding subjective evaluation of the sampled data set [25]. The support vector regression model is the supervised learning algorithm, which is mainly used to forecast the discrete values and follows the same principle as the SVM does. In SVR, the maximum number of points is present in the hyperplane best fit line. The purpose is to use the trained model to predict image quality.

Random forest is a method of learning comprehensive type theory. It is the machine learning technique that is used 
to rectify the problems in classification and regression, and it adopts the ensemble technique to combine several classifiers to deliver the solution for complex problems. Also, it consists of several decision trees to generate a more accurate and stable prediction. The final result is to combine all basic learning equipment with randomly sampled datasets through voting or averaging. Through the comprehensive vision of these machine learning methods, the accuracy and popularity of models can be effectively improved, and these models are difficult to simulate [26]. The basic learning tool used in the random forest is the CART decision tree. Classification and Regression Tree (CART) is a kind of classification algorithm, which builds the decision tree based on the Gini's impurity index as the splitting criterion. The nodes in the binary tree are split into two child nodes continuously. Then, the decision tree in the CART is used for predicting problems in classification or regression modeling. Its production characteristic is to randomly select a few characteristics, which fully guarantees the randomness of its characteristics. The training courses and the randomly selected forest decision trees are independent of each other and can be calculated on the following basis. Regarding the data in the training sample, each decision tree in the forest will judge it separately and take the most common result as its final product use. The random forest model can be used to determine the interaction between different features, regardless of the choice of features and their interactions [27]. This approach is very important and has many advantages. It is not easy to overfit and can be operated in parallel and calculated.

2.3.2. RF-IQA Method Flow. The RF-IQA method flow was shown in Figure 3. It played an important role in the development of multimedia applications, and the metric of QIA was stable with human perception during prediction. Based on the availability of the reference image, the quality image assessment was classified into three types such as reduced-reference QIA, no-reference QIA, and full-reference QIA [28, 29].

\section{Implementation and Effect Analysis of Earthworm Manure Bioengineering}

\subsection{Materials and Methods}

3.1.1. Materials. The experiment was carried out in an earthworm test base from July 3 to October 5, 2020, and the wheat residue was seasonally extracted from the farmland near the base.

3.1.2. Method. The area of the experimental micro-zone was $2 \mathrm{~m} \times 2 \mathrm{~m}$, and trenches were dug around for drainage. Spread nylon gauze at the bottom and around it to prevent earthworms from escaping, $40 \mathrm{~kg}$ of dry wheat straw was piled up, and earthworms are inoculated. The experiment included four treatment methods: T1: $2 \mathrm{~kg}$ of earthworms, adding $10 \mathrm{~kg}$ of dry Sludge in the center of the straw, T2: $1 \mathrm{~kg}$ of earthworms, and adding $10 \mathrm{~kg}$ of dry Sludge in the center of the straw; T3: $2 \mathrm{~kg}$ of earthworms without dry Sludge; T4: $1 \mathrm{~kg}$ of earthworms without dry Sludge; each treatment was repeated three times. During the experiment, water was poured every two days, and the humidity was controlled at about $70 \%$. Table 1 shows the basic characteristics of these test materials.

3.1.3. Determination. After the test was completed, the number of treated earthworms, noncorrosive decomposition residues, and earthworm manure + chemical manure were measured, and the nitrogen, phosphorus, and potassium content of the earthworm manure were measured. Total organic carbon was determined by dichromate oxidation, Total nitrogen was determined by the Kjeldahl method; total phosphorus was digested by $\mathrm{HF}-\mathrm{HCIO}_{4}$ and determined by molybdenum blue colorimetry method, and total potassium was determined by digestion with $\mathrm{HF}_{-} \mathrm{HCIO}_{4}$ and determined by flame photometry method.

\subsection{Analysis of Biological Experiment Effect}

3.2.1. The Material Composition and the Number of Earthworms after the Digestion of Wheat Straw. After the wheat straw was digested by earthworms, due to other physical, chemical, and biological influences, part of it becomes earthworm manure and other decomposition products, and some residues become waste [30]. Table 2 showed the amount of earthworm residues, manure, and wheat waste in the land after the earthworms digested the wheat stalks. From Table 2, it can be seen that the output of earthworms treated with mud has higher input than that of earthworms treated without mud. The soil has been treated with mud, and the yield of earthworms is also higher. The yield of earthworms treated by T1 is $2.54 \mathrm{~kg}$ per liter, and the yield of $1 \mathrm{~kg}$ of earthworms without a sludge layer was the lowest, $0.46 \mathrm{~kg}$ per liter. The amount of uncorroded and decomposed residues has obvious relationship with the existence of sewage sludge, and it also has a clear relationship with the inoculation level of earthworms [31]. The higher the inoculation rate of earthworms in the soil, the lower the number of residues that have not been decomposed by corrosion, and the production of earthworm manure in the soil is the highest. Because the addition of sludge leads to the content of nitrogen in the experimental treatment, which was beneficial to the growth of earthworms and the metabolism of microorganisms [32].

3.2.2. The Total Nitrogen, Phosphorus, and Potassium Content of the Material after the Earthworms Digested the Wheat Straw. Table 3 shows the nitrogen, phosphorus, and potassium content of vermicompost, which are all separated from rice straw. Earthworms decompose wheat residues and increase the total nitrogen of earthworm manure in the mud. $\mathrm{T} 2$ has the highest nitrogen content of $0.995 \%$, and the T4 treatment without sludge was $0.867 \%$, which was the lowest. From the results, it can be seen that the main reason for the increase of total nitrogen content in vermicompost 


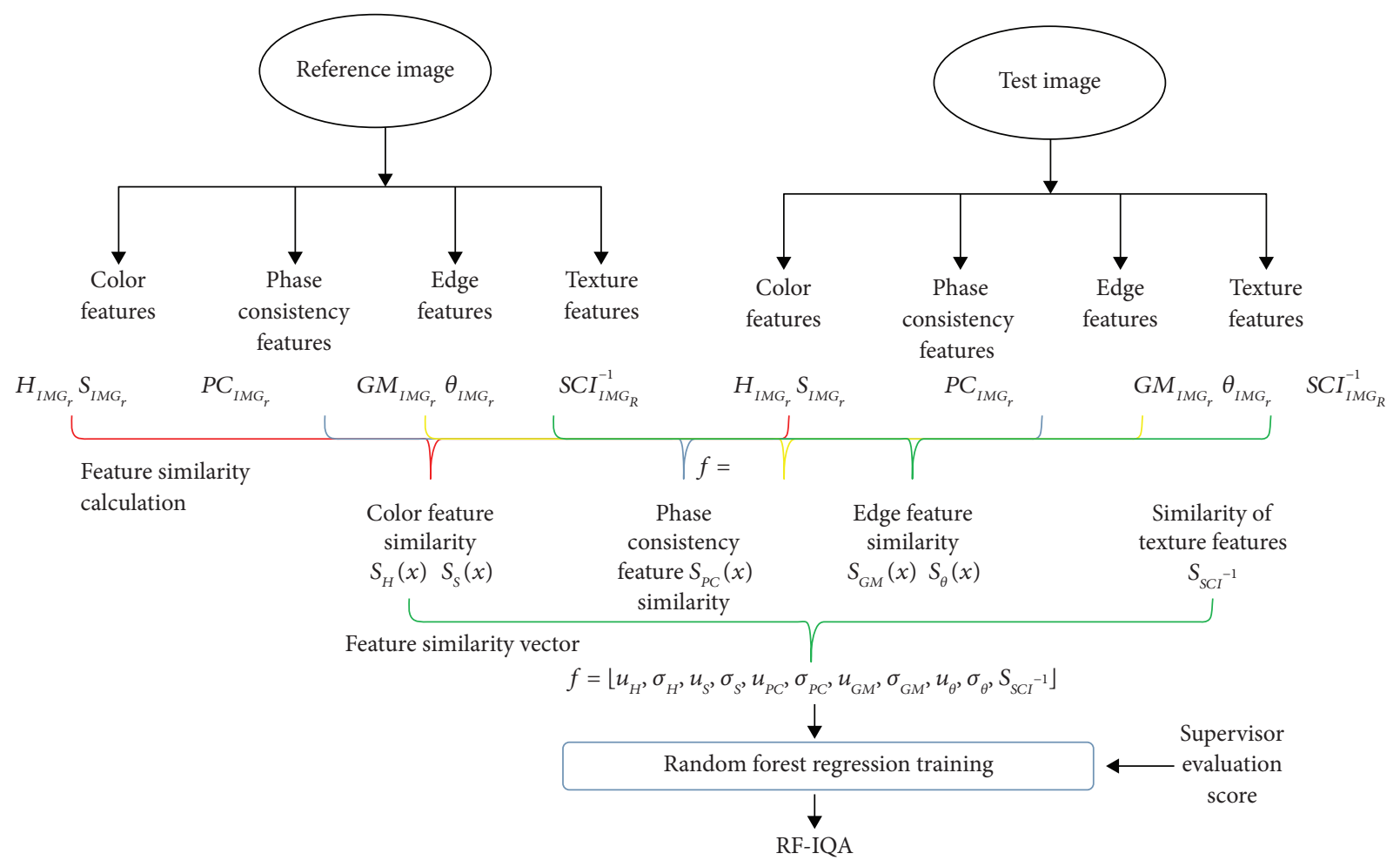

Figure 3: RF-IQA method flow.

TABLE 1: Basic physical and chemical properties of test materials.

\begin{tabular}{lcccc}
\hline Test materials & Total organic carbon (\%) & Total nitrogen (\%) & Total phosphorus (\%) & Total potassium (\%) \\
\hline Straw & 43.4 & 0.69 & 0.08 & 1.04 \\
Sludge & 34.04 & 2.76 & 1.45 & 0.78 \\
\hline
\end{tabular}

TABLE 2: The material composition and the number of earthworms after the digestion of wheat straw.

\begin{tabular}{|c|c|c|c|}
\hline \multirow{2}{*}{ Handle } & \multicolumn{3}{|c|}{ Determination object (kg/plot) } \\
\hline & Earthworm quantity & Nondecomposed straw & Vermicompost + decomposed matter \\
\hline $\mathrm{T} 1$ & $2.54 \pm 0.06$ & $1.82 \pm 0.37$ & $25.09 \pm 1.49$ \\
\hline $\mathrm{T} 2$ & $1.23 \pm 0.1$ & $3.25 \pm 0.47$ & $23.88 \pm 1.89$ \\
\hline T3 & $0.93 \pm 0.10$ & $4.27 \pm 0.56$ & $18.86 \pm 1.53$ \\
\hline $\mathrm{T} 4$ & $0.46 \pm 0.06$ & $5.36 \pm 0.41$ & $17.82 \pm 1.45$ \\
\hline
\end{tabular}

TABLE 3: The content of total nitrogen, phosphorus, and potassium of vermicompost.

\begin{tabular}{lccc}
\hline \multirow{2}{*}{ Handle } & \multicolumn{3}{c}{ Nutrient content $(\%)$} \\
& Total nitrogen & Total phosphorus & Total potassium \\
\hline T1 & $0.956 \pm 0.12$ & $0.166 \pm 0.015$ & $1.315 \pm 0.21$ \\
T2 & $0.995 \pm 0.21$ & $0.144 \pm 0.019$ & $1.385 \pm 0.17$ \\
T3 & $0.883 \pm 0.14$ & $0.116 \pm 0.017$ & $1.235 \pm 0.16$ \\
T4 & $0.867 \pm 0.14$ & $0.124 \pm 0.009$ & $1.277 \pm 0.27$ \\
\hline
\end{tabular}

compared with straw was that, in the process of growth and reproduction, on the one hand, earthworms absorb nitrogen from the material to meet the needs of their growth and reproduction and then return to the material through excreta (mainly mucus protein, ammonia, urea, uric acid, and allantoin). On the other hand, earthworms increase the active area of microorganisms to the material by feeding and crushing straw, promoting the mineralization of nitrogen, and promoting the transformation from $\mathrm{NH}_{4}-\mathrm{N}$ to $\mathrm{NO}_{3}-\mathrm{N}$ in available nitrogen [33]. The changing trend of total phosphorus and total potassium in vermicompost was the same, which was higher in the treatment of adding sludge. The content of total phosphorus was the highest in $\mathrm{T} 1$ treatment, reaching $0.166 \%$, and the content of total potassium was the highest in T2 treatment, up to $1.385 \%$.

Table 4 shows the nitrogen, phosphorus, and potassium content of wheat straw after earthworms' digestion. The results showed that the total nitrogen content of $\mathrm{T} 1$ and $\mathrm{T} 2$ treatments was higher than that of T3 and T4, and the highest content of $\mathrm{T} 1$ was $0.937 \%$. The reason for the high 
TABLE 4: The content of total nitrogen, phosphorus, and potassium of wheat straw after digestion.

\begin{tabular}{lccc}
\hline Handle & Total nitrogen & $\begin{array}{c}\text { Nutrient content (\%) } \\
\text { Total phosphorus }\end{array}$ & Total potassium \\
\hline T1 & $0.937 \pm 0.13$ & $0.106 \pm 0.008$ & $1.078 \pm 0.61$ \\
T2 & $0.915 \pm 0.12$ & $0.093 \pm 0.013$ & $1.094 \pm 0.52$ \\
T3 & $0.843 \pm 0.11$ & $0.085 \pm 0.011$ & $1.11 \pm 0.64$ \\
T4 & $0.824 \pm 0.14$ & $0.087 \pm 0.008$ & $1.125 \pm 0.59$ \\
\hline
\end{tabular}

TABLE 5: The content of organic matter in vermicompost and decomposition.

\begin{tabular}{lcr}
\hline Handle & \multicolumn{2}{c}{ Organic matter content (\%) } \\
T1 & Vermicompost + decomposition & Undecomposed straw \\
T2 & $33.32 \pm 2.76$ & $42.4 \pm 2.44$ \\
T3 & $34.42 \pm 1.84$ & $42.3 \pm 2.59$ \\
T4 & $35.52 \pm 2.54$ & $42.15 \pm 2.38$ \\
\hline
\end{tabular}
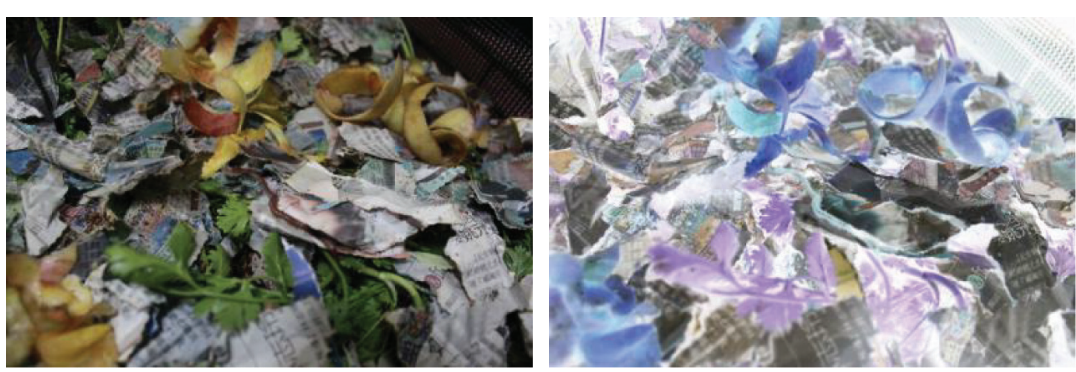

(a)
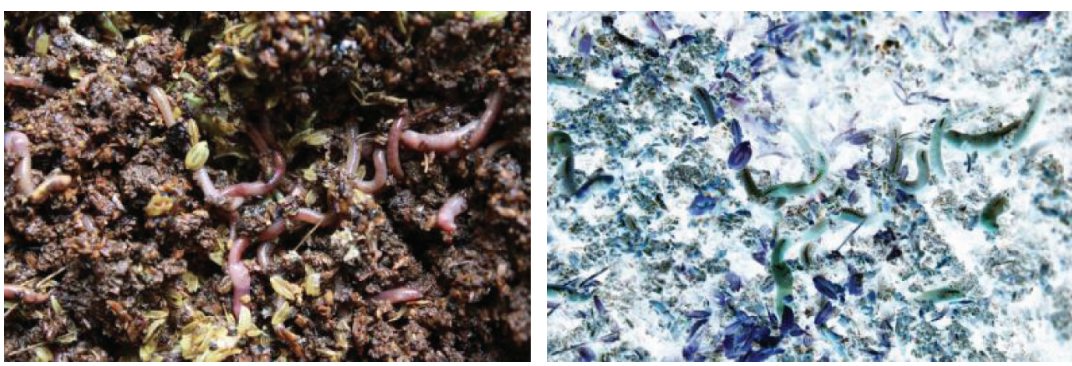

(b)
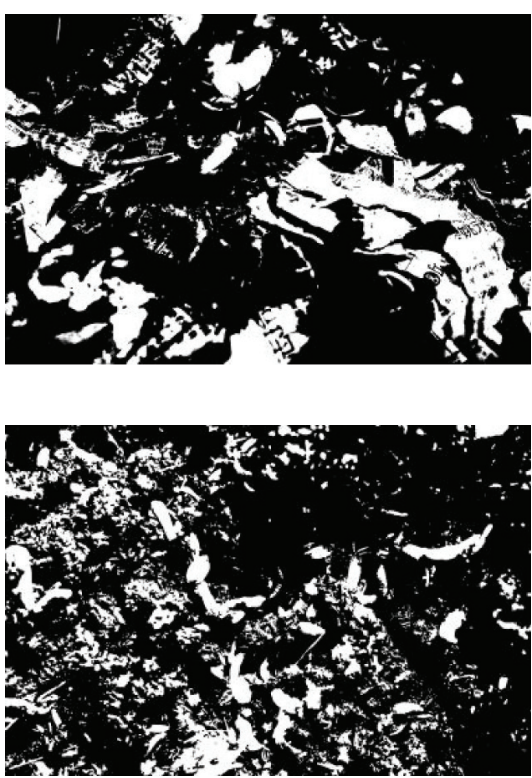

FIGURE 4: Image feature extraction and analysis in different stages of earthworm feces bioengineering. (a) The initial stage of vermicompost bioengineering. (b) Mid-to-late stage of vermicompost bioengineering.

TABle 6: Performance comparison of different regression methods.

\begin{tabular}{|c|c|c|c|c|}
\hline Database & Evaluating indicator & GRNN & SVR & $\mathrm{RF}$ \\
\hline \multirow{4}{*}{ TID2013 (3000) } & PLCC & 0.9147 & 0.8491 & 0.9236 \\
\hline & SROCC & 0.9024 & 0.8513 & 0.9167 \\
\hline & KROCC & 0.7485 & 0.6925 & 0.7653 \\
\hline & RMSE & 0.5738 & 0.5873 & 0.5694 \\
\hline \multirow{4}{*}{ TID2008 (1700) } & PICC & 0.9018 & 0.8269 & 0.9217 \\
\hline & SROCC & 0.8992 & 0.8183 & 0.9186 \\
\hline & KROCC & 0.7572 & 0.7094 & 0.7823 \\
\hline & RMSE & 0.5913 & 0.6779 & 0.5327 \\
\hline \multirow{4}{*}{ LIVE (779) } & PLCC & 0.9613 & 0.9783 & 0.9725 \\
\hline & SROCC & 0.9562 & 0.8765 & $0.9 \mathrm{~b} 74$ \\
\hline & KROCC & 0.8168 & 0.6792 & 0.8535 \\
\hline & RMSE & 6.9783 & 9.3154 & 6.0980 \\
\hline \multirow{4}{*}{ CSIQ (866) } & PLCC & 0.9513 & 0.8917 & 0.9536 \\
\hline & SROCC & 0.9589 & 0.8985 & 0.9542 \\
\hline & KROCC & 0.8357 & 0.7358 & 0.8472 \\
\hline & RMSE & 0.0593 & 0.1138 & 0.0621 \\
\hline
\end{tabular}



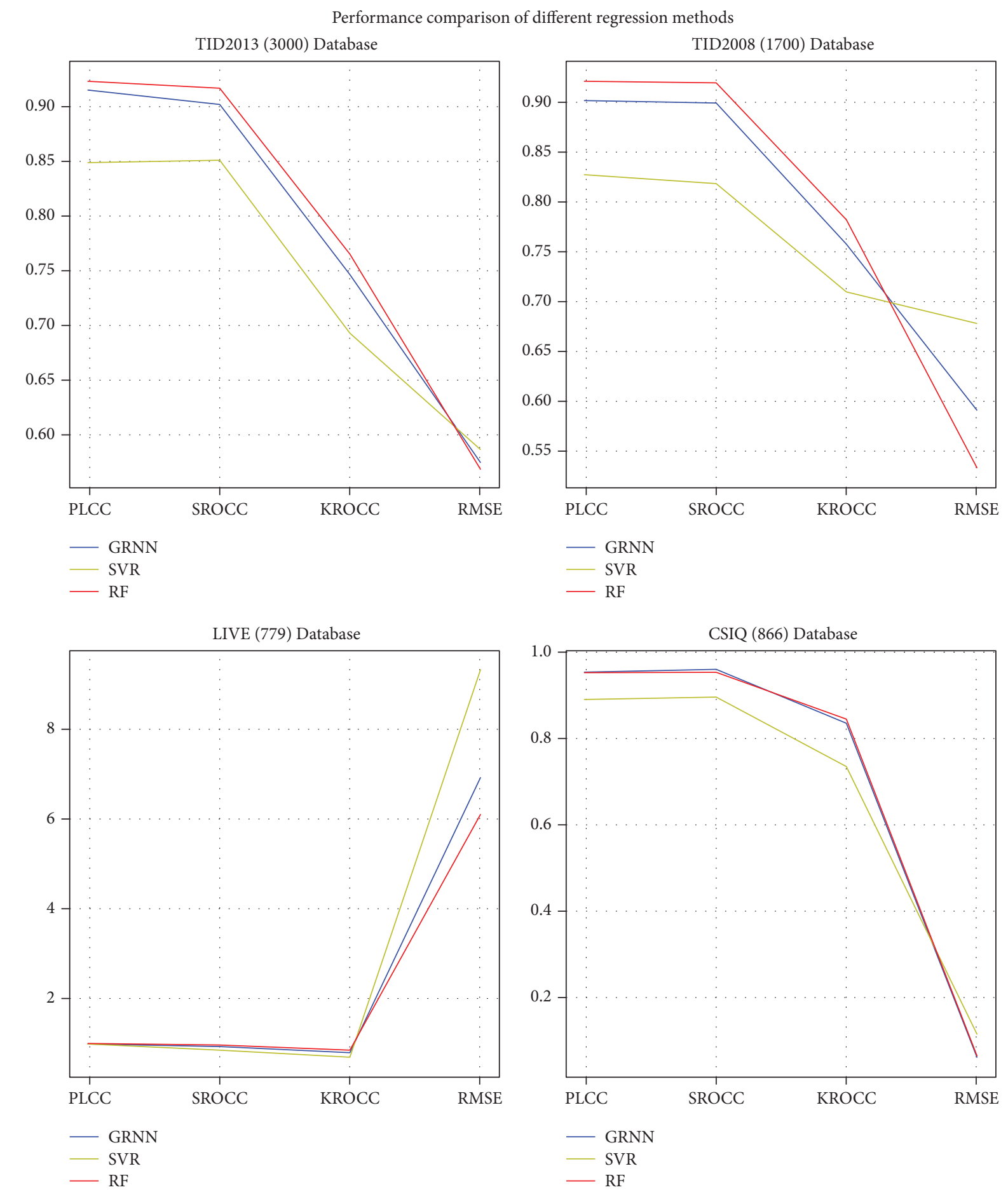

FIGURE 5: Specific image characteristics of different regression methods.

content of total nitrogen in straw treatments was that the content of total nitrogen in sludge was more than 4 times that of straw, which provides part of the nitrogen source for the growth of earthworms, and the earthworms themselves discharge body fluids in vitro, making nitrogen elements attached to the straw. The maximum content of total phosphorus in $\mathrm{T} 1$ treatment was $0.106 \%$, and the lowest in $\mathrm{T} 3$ treatment was $0.085 \%$. The content of total potassium was the highest in T4 treatment and the lowest in T1 treatment. The content of total potassium in the treatment with sludge was lower than that in the treatment without sludge. The content of nitrogen, phosphorus, and potassium increased in the treatment of decomposed straw, mainly due to the mineralization of organic matter, the decrease of straw quality, and the concentration of materials [34]. As a result, the content of nutrient elements per unit material was increased. 
TABLE 7: Performance comparison of RF-IQA method on different databases.

\begin{tabular}{|c|c|c|c|c|c|c|}
\hline Database & Evaluating indicator & PSNR & VSNR & SSIM & FSIM & RF-IQA \\
\hline \multirow{4}{*}{ TID2013 (3000) } & PLCC & 0.7017 & 0.7402 & 0.7895 & 0.8589 & 0.9236 \\
\hline & SROCC & 0.6396 & 0.6812 & 0.7417 & 0.8015 & 0.9167 \\
\hline & KROCC & 0.4698 & 0.5084 & 0.5588 & 0.6289 & 0.7653 \\
\hline & RMSE & 0.8832 & 0.8392 & 0.7608 & 0.6349 & 0.5694 \\
\hline \multirow{4}{*}{ TID2008 (1700) } & PICC & 0.5734 & 0.6820 & 0.7732 & 0.8738 & 0.9217 \\
\hline & SROCC & 0.5531 & 0.7046 & 0.7749 & 0.8805 & 0.9186 \\
\hline & KROCC & 0.4027 & 0.5340 & 0.5768 & 0.6946 & 0.7823 \\
\hline & RMSE & 1.0994 & 0.9815 & 0.8511 & 0.6525 & 0.5327 \\
\hline \multirow{4}{*}{ LIVE (779) } & PLCC & 0.8723 & 0.9231 & 0.9449 & 0.9597 & 0.9725 \\
\hline & SROCC & 0.8756 & 0.9274 & 0.9479 & 0.9634 & 0.9674 \\
\hline & KROCC & 0.6865 & 0.7616 & 0.7963 & 0.8337 & 0.8535 \\
\hline & RMSE & 13.3597 & 10.5059 & 8.9455 & 7.6780 & 6.0980 \\
\hline \multirow{4}{*}{ CSIQ (866) } & PLCC & 0.8000 & 0.8002 & 0.8613 & 0.9120 & 0.9536 \\
\hline & SROCC & 0.8058 & 0.8106 & 0.8756 & 0.9242 & 0.9542 \\
\hline & KROCC & 0.6084 & 0.6247 & 0.6907 & 0.7567 & 0.8472 \\
\hline & RMSE & 0.1575 & 0.1575 & 0.1334 & 0.1077 & 0.0621 \\
\hline
\end{tabular}

3.2.3. The Organic Matter Content of the Material after the Earthworms Digested Wheat Straw. Table 5 shows the organic carbon content in the material after treating the wheat residue with earthworms. We found that the content of organic carbon decreased in all experiment treatments, which reflected the decomposition of organic matter by earthworms. In the process of decomposition of organic matter, part of the organic carbon was released in the form of $\mathrm{CO}_{2}$, resulting in a decrease in the content of organic carbon in the mixture. The decrease of organic carbon content was related to the activity intensity of earthworms: in the combinations with better and more active growth of earthworms, the decomposition ability of earthworms was stronger, and the content of organic carbon decreases more greatly. This can explain the lower content of organic carbon in straw treatment with sludge.

\subsection{Image Feature Analysis during Biological Digestion}

3.3.1. Experimental Method. In the experiment, the K-Plexion cross-check method was used to build a regression model based on machine learning [35]. The regression model was used to examine the correlation among two or more variables to determine the variables based on other variables. In other words, regression analysis was the predictive modeling technique that was used to find the variation between dependent and independent variables. It is mainly used for time series modeling, finding causal effects between variables, and prediction. The K-Plexion crosscheck method was a more powerful model evaluation method, which avoids model duplication to a certain extent. The K-Plexion cross-check method was used to negotiate the duplication of the models with an efficient evaluation method. The implementation process of the cross-check method was divided into the following stages: try to use nonrepetitive samples in the test and training groups to avoid repeated concentration of sample data.

(1) Through a nonrepetitive sampling, the data set was randomly divided into K groups; (2) a K-1 data group was used to study the training model, and the rest were used as an experimental data group for testing; (3) the second stage was repeated, corresponding models and evaluations were obtained, and the results were summarized; (4) finally, repeat these three steps to get the average result $\mathrm{N}$ and reduce the error in data decomposition.

\subsubsection{Comparative Experiments of Different Machine} Learning Regression Methods. Feature extraction reduced the number of features present in the data by creating new data datasets based on existing data, then the new data set features to have the ability to analyze most of the information present in the original set of features. Through the image feature extraction technology, combined with the different stages of earthworm feces bioengineering, the main images were obtained as shown in Figure 4.

Table 6 listed the performance indicators of each method. The higher the values of PLCC, SROCC, and KROCC, the more effective the image quality evaluation method. It can be seen that the random forest method is more successful than the generalized neural regression network and the method of learning and training regression models. Meanwhile, in this experiment, a random forest method was selected to learn and train regression models and to link image characteristics with image quality. The specific image characteristics of the data in Table 6 were shown in Figure 5.

During the experiment, we compared the GRNN, SVR, and RF methods. In each database, we conducted experiments using the same cross-check method.

3.3.3. Overall Performance Comparison. To further test and verify the performance of the RF-IQA method in the image quality evaluation, the seven standard complete reference image quality evaluation methods were compared and analyzed. In each database, using the average result of repeated trials as the experimental value of RF-IQA, we listed the performance of each image quality evaluation method in 
Performance comparison of RF-IQA method on different data
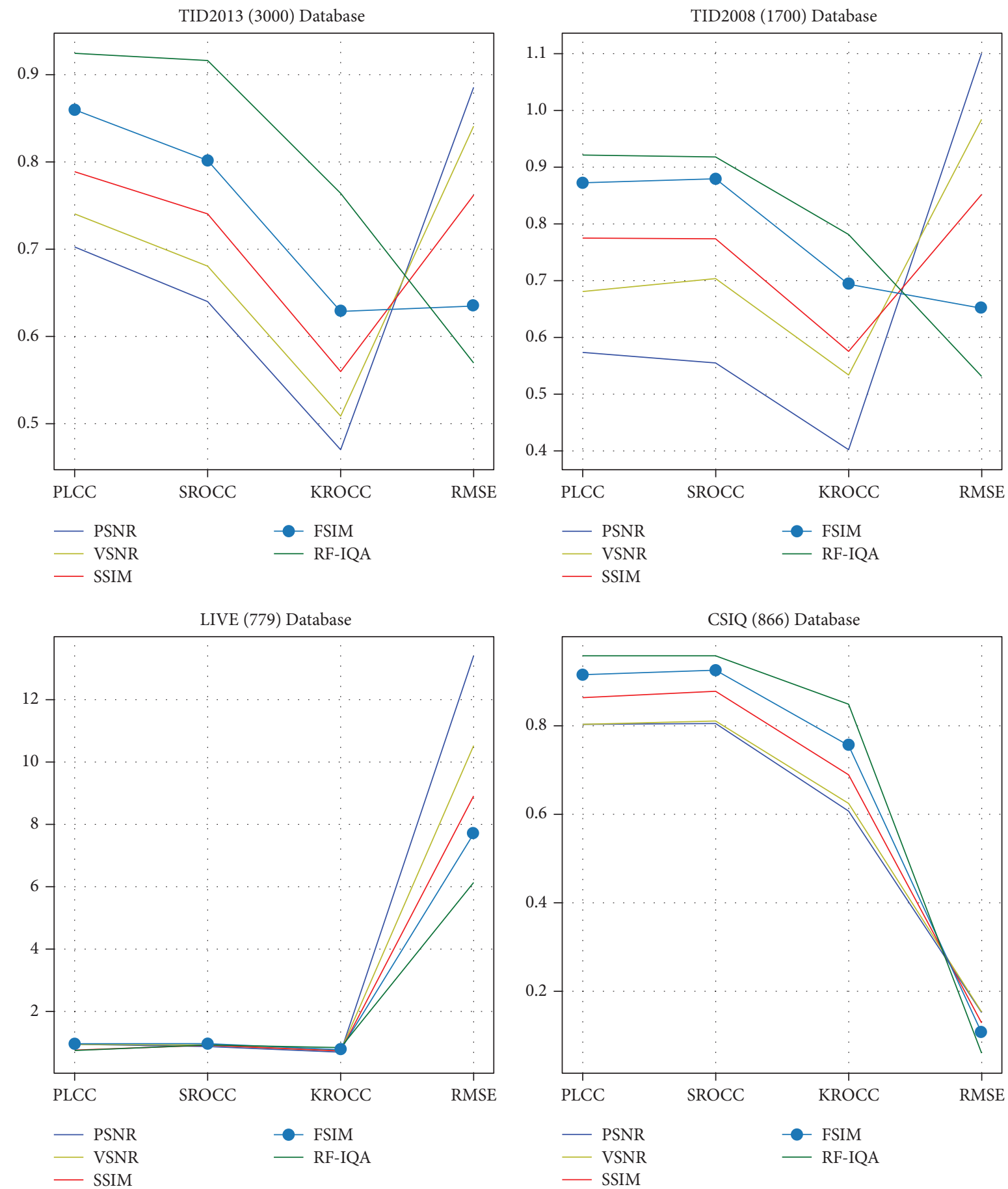

Figure 6: Performance comparison of RF-IQA method on different databases.

each database and calculated the respective values of SROC, KROC, PLCC, and RMSE.

As shown in Table 7, the proposed RF-IQA image quality evaluation method has obvious advantages, because the performance indicators were at the forefront of the database and higher than most traditional image quality evaluation methods. The purpose of this method was to enable users to better understand the quality of the image. The specific image characteristics of the data in Table 7 are shown in Figure 6.

As shown in Table 8, we calculated the average and weighted average of different image quality evaluation methods according to Table 7, which shows that the overall performance of the RF-IQA method is better than the other seven traditional evaluation methods. 
TABLE 8: The overall performance comparison of the RF-IQA method in the four major databases.

\begin{tabular}{lcccccc}
\hline Comprehensive performance & Evaluation index & PSNR & VSNR & SSIM & FSIM & RF-IQA \\
\hline & PLCC & 0.7369 & 0.7864 & 0.8422 & 0.9011 & 0.9429 \\
Avg. & SROCC & 0.7185 & 0.7810 & 0.8350 & 0.8924 & 0.9392 \\
& KROCC & 0.5419 & 0.6072 & 0.6557 & 0.7285 & 0.8121 \\
& RMSE & 3.8750 & 3.1210 & 2.6727 & 2.2683 & 1.8156 \\
\hline \multirow{3}{*}{ W.Avg. } & PLCC & 0.7017 & 0.7553 & 0.8140 & 0.8825 & 0.9332 \\
& SROCC & 0.6681 & 0.7354 & 0.7942 & 0.8593 & 0.9286 \\
& KROCC & 0.4973 & 0.5622 & 0.6108 & 0.6891 & 0.7919 \\
& RMSE & 2.3739 & 1.9711 & 1.7042 & 1.4324 \\
\hline
\end{tabular}

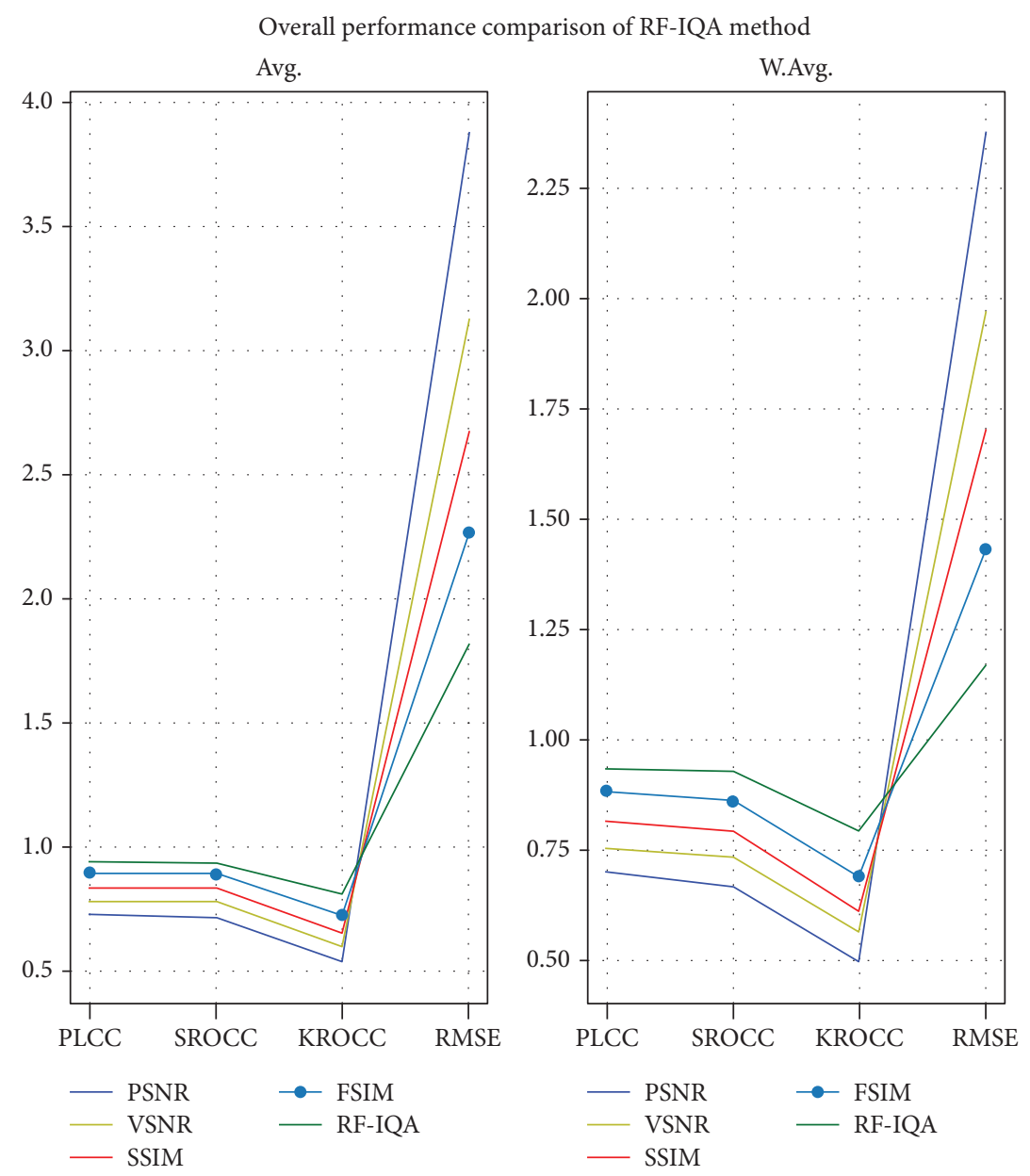

FIgURE 7: The overall performance comparison of the RF-IQA method in the four major databases.

Through the RF-IQA method, we analyzed the appearance features involved in the image quality assessment research, then analyzed the impact of the color and texture characteristics of the image on the image quality, and used the image quality-related extraction methods. The image characteristics of the data in Table 8 are shown in Figure 7.

The RF-IQA method started from the extraction of the reference image, tested the phase consistency, boundary characteristics, color characteristics, and texture characteristics of the image respectively, and then calculated the similarity of the corresponding characteristics, created a vector of similar characteristics, and finally used the random forest machinery learning to know the regression training mode. It established a relationship between features and image quality and compared and evaluated image quality [36]. The image quality evaluation method proposed in this article has better performance and was more suitable for human vision and cognitive characteristics.

\section{Conclusion}

Earthworms can decompose the organic matter in the soil into a simple organic matter, even inorganic matter, and can better enter the next round of the biological cycle. The residues of crops contain a large amount of organic matter, which can be combined with other biological waste 
materials, such as sewage sludge in different proportions, which can be used as bait for earthworms. The test results show that adding a certain amount of mud helps the earthworms better deal with the residues in the soil. Except for the small amount of uncorroded residues left in the dry straw pile, the residues in the pile are completely processed by earthworms. Because the pile of straw is too dry, the earthworms cannot digest them, and the nutrient shortage in the pile causes the earthworms to escape from the pile to the ground. The effective nutrient content in sewage sludge is very high, especially the nitrogen content. After mixing the mud, the relationship between carbon and nitrogen can be better adjusted, so that the earthworms can obtain food. This article analyzes the quality evaluation stage of the complete reference image from different angles, to better monitor the impact of the experiment, and provides a quality evaluation report on the reference image of the experiment. Using indepth learning methods and machine learning methods to improve the performance of image quality evaluation in the image feature extraction stage and image quality mapping stage, and to improve image quality, will improve the control of bioengineering and improve management efficiency.

\section{Data Availability}

The data used to support the findings of this study are included within the article.

\section{Conflicts of Interest}

The authors declare that they have no conflicts of interest.

\section{References}

[1] R. Joshi, J. Singh, and A. P. Vig, "Vermicompost as an effective organic fertilizer and biocontrol agent: effect on growth, yield and quality of plants," Reviews in Environmental Science and Biotechnology, vol. 14, no. 1, pp. 137-159, 2015.

[2] S. Gopalakrishnan, S. Vadlamudi, P. Bandikinda et al., "Evaluation of Streptomyces strains isolated from herbal vermicompost for their plant growth-promotion traits in rice," Microbiological Research, vol. 169, no. 1, pp. 40-48, 2014.

[3] N. Q. Arancon, C. A. Edwards, E. N. Yardim, T. J. Oliver, R. J. Byrne, and G. Keeney, "Suppression of two-spotted spider mite (Tetranychus urticae), mealy bug (Pseudococcus $\mathrm{sp}$ ) and aphid (Myzus persicae) populations and damage by vermicomposts," Crop Protection, vol. 26, no. 1, pp. 29-39, 2007.

[4] J. Pathma and N. Sakthivel, "Microbial diversity of vermicompost bacteria that exhibit useful agricultural traits and waste management potential," SpringerPlus, vol. 1, pp. 1-19, 2012.

[5] Y. Simsek Ersahin, K. Haktanir, and Y. Yanar, "Vermicompost suppresses Rhizoctonia solani Kühn in cucumber seedlings," Journal of Plant Diseases and Protection, vol. 116, no. 4, pp. 182-188, 2009.

[6] V. Prabal, SK. Sood, and S. Kalra, "Cloud-centric IoT based student healthcare monitoring framework," Journal of Ambient Intelligence and Humanized Computing, vol. 9, no. 1, pp. 1293-1309, 2018.

[7] M. Renuka and J. Shyla, "Analysis of various data mining techniques to predict diabetes mellitus," International Journal of Applied Engineering Research, vol. 11, no. 1, pp. 727-730, 2016.

[8] J. S. Rumsfeld, K. E. Joynt, and T. M. Maddox, "Big data analytics to improve cardiovascular care: promise and challenges," Nature Reviews Cardiology, vol. 13, no. 6, pp. 350-359, 2016.

[9] S. Sanjay, S. K. Sood, and S. K. Gupta, "IoT-based cloud framework to control Ebola virus outbreak," Journal of Ambient Intelligence and Humanized Computing, vol. 9, no. 3, pp. 459-476, 2018.

[10] C. Stergiou, K. E. Psannis, B.-G. Kim, and B. Gupta, "Secure integration of IoT and cloud computing," Future Generation Computer Systems, vol. 78, no. 6, pp. 964-975, 2018.

[11] D. B. Alencar, C. M. Affonso, R. C. L. Oliveira, and J. C. R. Filho, "Hybrid approach combining SARIMA and neural networks for multi-step ahead wind speed forecasting in Brazil," IEEE Access, vol. 6, no. 1, pp. 55986-55994, 2018.

[12] A. Alsarhan, A. Itradat, A. Y. Al-Dubai, A. Y. Zomaya, and G. Min, "Adaptive resource allocation and provisioning in multi-service cloud environments," IEEE Transactions on Parallel and Distributed Systems, vol. 29, no. 1, pp. 31-42, 2018.

[13] A.-M. Ammar, J. Luo, Z. Tang, and O. Wajdy, "Intra-balance virtual machine placement for effective reduction in energy consumption and SLA violation," IEEE Access, vol. 7, no. 5, pp. 72387-72402, 2019.

[14] N. S. Dey and T. Gunasekhar, "A comprehensive survey of load balancing strategies using Hadoop queue scheduling and virtual machine migration," IEEE Access, vol. 7, no. 3, pp. 92259-92284, 2019.

[15] A. Jerome, M. Yuksel, S. H. Ahmed, and M. Bassiouni, "SDNbased load balancing for multi-path TCP," in Proceedings of the IEEE INFOCOM 2018-IEEE Conference on Computer Communications Workshops (INFOCOM WKSHPS), pp. 859-864, Honolulu, HI, USA, April 2018.

[16] N. Nehra, R. B. Patel, and V. K. Bhat, "A framework for distributed dynamic load balancing in heterogeneous cluster," Journal of Computer Science, vol. 3, no. 1, pp. 14-24, 2007.

[17] K. Djemame, D. Armstrong, J. Guitart, and M. Macias, “A risk assessment framework for cloud computing," IEEE Transactions on Cloud Computing, vol. 4, no. 3, pp. 265-278, 2016.

[18] N.-T. Nguyen, B.-H. Liu, S.-I. Chu, and H.-Z. Weng, "Challenges, designs, and performances of a distributed algorithm for minimum-latency of data-aggregation in multichannel WSNs," IEEE Transactions on Network and Service Management, vol. 16, no. 1, pp. 192-205, 2019.

[19] L. B. Godfrey and M. S. Gashler, "Neural decomposition of time-series data for effective generalization," IEEE Transactions on Neural Networks and Learning Systems, vol. 29, no. 7, pp. 2973-2985, 2018.

[20] W. Hussain and O. Sohaib, “Analysing cloud QoS prediction approaches and its control parameters: considering overall accuracy and freshness of a dataset," IEEE Access, vol. 7, no. 4, pp. 82649-82671, 2019.

[21] V. Vijayakumar, M. K. Priyan, G. Ushadevi, R. Varatharajan, G. Manogaran, and P. V. Tarare, "E-health cloud security using timing enabled proxy Re-encryption," Mobile Networks and Applications, vol. 24, no. 3, pp. 1034-1045, 2018.

[22] C. Thota, R. Sundarasekar, and G. Manogaran, "Centralized fog computing security platform for IoT and cloud in healthcare system," in Exploring the Convergence of Big Data and the Internet of Things, pp. 141-154, IGI Global, Hershey, PA, USA, 2018. 
[23] J. Gao, H. Wang, and H. Shen, "Machine learning based workload prediction in cloud computing," in Proceedings of the 2020 29th International Conference on Computer Communications and Networks (ICCCN), Honolulu, HI, USA, August 2020.

[24] H. Bolívar, M. Martínez, R. González, and O. Sanjuán, "Complexity analysis of a matchmaker based on hidden Markov model for decentralised grid scheduling," International Journal of Grid and Utility Computing, vol. 5, no. 3, p. 190, 2014.

[25] A. Hussain, M. Aleem, and A. Khan, "RALBA: a computationaware load balancing scheduler for cloud computing," Cluster Computing, vol. 21, no. 5, pp. 1667-1680, 2018.

[26] W. Hussain, F. K. Hussain, O. Hussain, R. Bagia, and E. Chang, "Risk-based framework for SLA violation abatement from the cloud service provider's perspective," The Computer Journal, vol. 61, no. 9, pp. 1306-1322, 2018.

[27] FA. Khan, S. Shaheen, and M. Asif, "Towards reliable and trustful personal health record systems: a case of cloud-dew architecture based provenance framework," Journal of Ambient Intelligence and Humanized Computing, vol. 10, no. 1, pp. 3795-3808, 2019.

[28] X. Yan, S. Wang, A. A. A. El-Latif, and X. Niu, "Visual secret sharing based on random grids with abilities of AND and XOR lossless recovery," Multimedia Tools and Applications, vol. 74, no. 9, pp. 3231-3252, 2013.

[29] A. A. Abd El-Latif and X. Niu, "A hybrid chaotic system and cyclic elliptic curve for image encryption," AEU - International Journal of Electronics and Communications, vol. 67, no. 2, pp. 136-143, 2013.

[30] P. Kumar and R. Kumar, "Issues and challenges of load balancing techniques in cloud computing," ACM Computing Surveys, vol. 51, no. 6, pp. 1-35, 2019.

[31] K. Huang, FS. Li, and YF. Wei, "Changes of bacterial and fungal community compositions during vermicomposting of vegetable wastes by Eisenia foetida," Bioresource Technology, vol. 150, pp. 235-241, 2013.

[32] NQ. Arancon, PA. Galvis, and CA. Edwards, "Suppression ofinsect pest populations and damage to plants by vermicomposts[J]," Bioresource Technology, vol. 96, pp. 1137-1142, 2005.

[33] M. Aira, F. Monroy, and J. D. Eisenia, "Fetida (Oligochaeta, Lumbricidae) activates fungal growth, triggering cellulose decomposition during vermicomposting," Microbial Ecology, vol. 52, no. 4, pp. 738-747, 2006.

[34] S. Bansal and K. K. Kapoor, "Vermicomposting of crop residues and cattle dung with Eisenia foetida," Bioresource Technology, vol. 73, pp. 95-98, 2000.

[35] Y. Zhang, H. Ma, and S. Zhao, "Assessment of hydropower sustainability: review and modeling," Journal of Cleaner Production, vol. 321, p. 128898, 2021.

[36] M. Shen, Y. Zhang, H. Xu, and H. Ma, "MOFs based on the application and challenges of Perovskite Solar Cells," iScience, vol. 24, no. 9, p. 103069, 2021. 